\title{
Response Preparation, Response Conflict, and the Effects of Irrelevant Flanker Stimuli
}

\author{
Peter Wühr ${ }^{1}$ and Herbert Heuer ${ }^{2}$ \\ 'Institut für Psychologie, Technische Universität Dortmund, Germany \\ ${ }^{2}$ Leibniz-Institut für Arbeitsforschung, Dortmund, Germany
}

ABSTRACT

The present study explored how response preparation modulates the effects of response conflict as induced by irrelevant flanker stimuli. In Experiments 1 and 2, an unreliable response cue (i.e., valid in $75 \%$ of trials but invalid in $25 \%$ of trials) preceded the stimulus display containing a target stimulus and different types (i.e., identical, neutral, compatible, or incompatible) flanker stimuli. In Experiment 3, a fully reliable response cue (i.e., valid in 100\% of trials) or a neutral cue preceded the stimulus display. There were two major findings. First, valid response cues always improved performance in terms of speed and accuracy when compared to invalid or neutral cues, indicating that the cues were used to selectively prepare the indicated response. Second, response preparation with unreliable response cues did not modulate flanker-induced response conflict in reaction times (RTs; and not consistently in error percentages), whereas response preparation with reliable cues eliminated flanker-induced response conflict. According to these results, only extreme levels of response preparation modulate (flanker-induced) response conflict. The results of computer simulations suggest some boundary conditions for our conclusion.

Eriksen task, flanker task, response cueing, response preparation

\section{Response Conflict and Selective Response Preparation}

Objects in our environment afford different and often incompatible actions. A major task of cognitive control is to resolve resulting conflicts by selecting those responses that serve our current goals and by suppressing competing responses (e.g., Botvinick, Braver, Barch, Carter, \& Cohen, 2001; Norman \& Shallice, 1986). As an example, consider a soccer player who is in possession of the ball and looks for a team mate he could pass the ball to. A response conflict would arise if two players, a team mate on the left side and an opponent on the right side, are simultaneously waving to receive the ball. In this situation, the player would be supposed to select a pass to the left side, which would be a correct response. The pass to the right side, that is, to the player of the opposing team, would be an incorrect response. How would the timing and the accuracy of the pass be affected if the player heard someone shout "pass to the right" before noting the two players who could receive the pass? The purpose of the present study is to determine how selective response preparation that produces an a priori bias to produce the correct or the incorrect response affects the latency and the accuracy of responses in a task with competing response tendencies.
Response preparation can be generalized, so that all possible responses are speeded up (e.g., Falkenstein, Hohnsbein, Hoormann, \& Kleinsorge, 2003), or it can be selective, so that only one (or a subset) of the alternative responses is prepared. Selective response preparation is the focus of the present experiments. It is often induced by response cues that predict the correct response to the next stimulus with a specific probability (e.g., Leuthold, Sommer, \& Ulrich, 1996). In a choice task with only two possible responses, one of the alternative responses is cued, and this response will be required in the majority of trials (e.g., 75 or $80 \%$ trials with valid cues) but not in all trials (e.g., 25 or $20 \%$ of trials with invalid cues). Thereby, not only reaction time (RT) of the prepared response can be analyzed but also RT of the unprepared response (e.g., Rosenbaum \& Kornblum, 1982). The typical result of selective response preparation is faster RT and smaller error rate of the

Corresponding author: Peter Wühr, Technische Universität Dortmund, Institut für Psychologie, Emil-Figge-Strasse 50, 44227, Dortmund, Germany. Phone: 0049-231-755-7928.Email: peter.wuehr@tu-dortmund.de 
prepared response. Rather than by response cues, selective preparation has also been induced by a higher relative frequency of one of the alternative responses (e.g., Bertelson \& Tisseyre, 1966; Dillon, 1966; Heuer, 1982; LaBerge \& Tweedy, 1964).

The effects of selective response preparation on performance are nicely captured by sequential-sampling models. These models posit a continuous noisy activation of response codes by the stimulus presented in a certain trial; the activation of the response codes can be conceived as evidence in favor of the associated stimuli being presented. The response is initiated when either activation of one code (cf. Vickers, 1979) or the difference between the activations of different codes (cf. Laming, 1968) reaches a threshold (cf. Smith \& Ratcliff, 2004, for an overview of sequential-sampling models). Selective preparation can be modelled by preactivation of one of the alternative responses. This modelling is consistent with electrophysiological findings which revealed preactivation of a cued response at a cortical level in terms of the lateralized readiness potential (e.g., Wauschkuhn, Wascher, \& Verleger, 1997) or lateralized event-related beta desynchronization (e.g., Doyle, Yarrow, \& Brown, 2005). Theoretically, preactivation of response codes biases response selection so that $\mathrm{RT}$ is faster and error rate is smaller when the prepared response is the correct one as compared to trials in which the unprepared response is the correct one, and this prediction matches the experimental findings (e.g., Smith \& Ratcliff, 2004).

Sequential-sampling models of response selection can include competition between response codes in the form of mutual inhibition (Heuer, 1987; Usher \& McClelland, 2001). This is particularly the case for models applied to conflict paradigms such as the flanker task or the Simon task (Cohen, Servan-Schreiber, \& McClelland, 1992; Zhang, Zhang, \& Kornblum, 1999; Zorzi \& Umiltà, 1995). From the perspective of this type of model, the higher the activation of an incorrect response code by irrelevant stimuli or stimulus features, the more inhibition it should exert on the correct response code, and the longer it should take to select the correct response. More specifically, if the correct response is the prepared one, the activation of the alternative response code should be only weak, and the effect of response conflict should be small. In contrast, if the correct response is the unprepared one, the effect of response conflict, originating from the incorrect and strongly activated response code, should be large. Thus, one would expect smaller effects of response conflict on prepared responses than on unprepared ones (cf. Buckolz, Stapleton, \& Alain, 1994; Wascher \& Wolber, 2004).

\section{Selective Response Preparation and Response Conflict in the Simon Task}

The impact of selective response preparation on the effects of response conflict has been studied almost exclusively in the Simon task. In that task, participants produce spatially defined responses to a nonspatial stimulus feature such as color. The variation of irrelevant stimulus location produces spatially corresponding conditions, in which stimulus and response locations match, and spatially noncorresponding conditions, in which stimulus and response locations mismatch. Shorter
RT in spatially corresponding than in noncorresponding conditions constitutes the Simon effect (e.g., Simon, 1969; Simon \& Rudell, 1967; for a review see Hommel, 2011).

The Simon effect is generally attributed to interference at the response-selection stage. Most accounts assume that stimulus location is automatically encoded and activates the spatially corresponding response code (e.g., Ansorge \& Wühr, 2004; Hommel, 1997; Kornblum, Hasbroucq, \& Osman, 1990; Zorzi \& Umiltà, 1995). In spatially corresponding conditions, irrelevant stimulus location coactivates the correct response and, therefore, facilitates its selection. In contrast, in spatially noncorresponding conditions, irrelevant stimulus location activates an incorrect response that competes for selection with the correct response.

The modulation of the effects of response conflict in the Simon task by selective preparation has been tested in a number of studies. Contrary to the expectations outlined above, the Simon effect was consistently found to be larger instead of smaller for prepared (cued) responses than for unprepared (uncued) responses (e.g., Proctor, Lu, \& Van Zandt, 1992; Verfaellie, Bowers, \& Heilman, 1988; Wascher \& Wolber, 2004; Wühr, 2006). However, this unexpected result might not originate at response selection, but the modulation of the effects of response conflict at that level of processing might be superposed and dominated by an effect of response cues or response preparation on stimulus processing.

Wascher and Wolber (2004) measured electrophysiological correlates of stimulus processing and response preparation in addition to behavioral data. Behaviorally, they observed the typical effects of response cues on RT and accuracy. Importantly, the electrophysiological data revealed effects of the response cues not only on response preparation, as indicated by the lateralized readiness potential, but also on attention and thus the efficiency of perceptual processing. The shift of attention to the cued side was indicated by the N2pc, a lateralized potential related to selective attention. The shift of spatial attention to the side of the cued (and prepared) response would increase the Simon effect with valid cues by facilitating stimulus processing in corresponding conditions and hampering stimulus processing in noncorresponding conditions. Conversely, with invalid response cues, the shift of spatial attention to the side of the cued response would decrease the Simon effect by hampering stimulus processing in corresponding conditions and facilitating stimulus processing in noncorresponding conditions. Currently, it is not fully clear whether the shift of attention is induced by the response cues (Buhlmann \& Wascher, 2006) or by response preparation per se (Wühr \& Heuer, 2015).

The hypothesis that the expected modulation of the effects of response conflict by response preparation is overridden by additional variations of the efficiency of stimulus processing in the Simon task is supported by findings obtained with fully reliable response cues. In the limiting case of (almost) perfect preparation, response selection becomes independent of the response-relevant stimuli and the task approaches a simple-RT task. Thus, the Simon effect should (almost) disappear. In fact, Wühr (2006) observed that fully reliable response cues reduce the Simon effect as compared to a condition without re- 
sponse cues. To uncover the expected modulation of response conflict by response preparation with unreliable response cues, in the present experiments, we use the flanker task that, in contrast to the Simon task, should be essentially insensitive to variations of stimulus processing that result from lateral attentional shifts.

\section{Response Conflict and the Flanker Paradigm}

The flanker paradigm (B. A. Eriksen \& Eriksen, 1974; C. W. Eriksen \& Eriksen, 1979; for review see C. W. Eriksen, 1995) is another established paradigm for investigating the effects of response conflict. In a typical flanker experiment, the stimulus set may consist of four letters. Two letters $(A$ and $B)$ are assigned to one response, and two other letters $(C$ and $D)$ are assigned to another response. Each stimulus display consists of a target letter at screen center and several distractor stimuli, the flankers. The flanker stimuli are typically all the same and distributed symmetrically around the target. There are three different targetflanker relations: (a) Identical flankers look the same as the target and require the same response (e.g., AAA), (b) compatible flankers look different from the target but require the same response (e.g., BAB), and (c) incompatible flankers look different from the target and require a different response (e.g., CAC). The typical pattern of results is fastest RT to targets with identical flankers, intermediate RT with compatible flankers, and longest RT with incompatible flankers (e.g., B. A. Eriksen \& Eriksen, 1974; C. W. Eriksen \& Eriksen, 1979; Fournier \& Eriksen, 1990; Taylor, 1977). In addition, there may be neutral flankers which are not assigned to a response. For them, RT is usually intermediate between conditions with compatible and incompatible flankers (e.g., B. A. Eriksen \& Eriksen, 1974; C. W. Eriksen \& Eriksen, 1979; Taylor, 1977). The impact of the flanker stimuli declines when their spatial separation from the target increases (e.g., B. A. Eriksen \& Eriksen, 1974), but significant effects have been obtained with spatial separations as large as $3^{\circ}$ (Fournier \& Eriksen, 1990) or even $5^{\circ}$ of visual angle (Miller, 1991).

C. W. Eriksen and Schultz (1979) proposed a continuous-flow model to account for the basic pattern of results observed with the flanker task. The model distinguishes different stages, such as a perceptualidentification stage and a response-selection stage. In this respect it is similar to discrete-stage models (cf. Sanders, 1980; Sternberg, 1969). It differs with respect to the assumption that the output of each stage is continuously fed into the subsequent stage (for a general discussion of this type of model see McClelland, 1979; for a comparison of both types of model see Sanders, 1990). In the framework of the continuous-flow model, faster RT for identical flankers (same stimuli, same response) than for compatible flankers (different stimuli, same response) is attributed to facilitation and inhibition, respectively, at a perceptual stage of processing (e.g., C. W. Eriksen \& Schultz, 1979; Fournier \& Eriksen, 1990). Faster RT for compatible flankers (different stimuli, same response) than for incompatible flankers (different stimuli, different responses) is attributed to facilitation and inhibition, respectively, at a response-selection stage (e.g., C. W. Eriksen, 1995; C. W. Eriksen \& Schultz, 1979).
The involvement of response selection in the flanker effect can be evidenced both at the cortical level (from the lateralized readiness potential) and at the peripheral level (from the electromyogram). At both levels, activation of the incorrect response can be observed that is stronger in incompatible than in compatible trials (e.g., Coles, Gratton, Bashore, Eriksen, \& Donchin, 1985; Gratton, Coles, Sirevaag, Eriksen, \& Donchin, 1988; Smid, Mulder, \& Mulder, 1990; Verleger, Kuniecki, Möller, Fritzmannova, \& Siebner, 2009). In contrast to the Simon task, as long as flankers are symmetrically distributed around the centrally located target stimulus. shifts of spatial attention towards the side of the cued response should not (or only marginally) modulate the efficiency of stimulus processing. With this configuration, attentional shifts to the left or right would both go along with attending to a flanker. Therefore, potential modulations of the efficiency of stimulus processing at different locations should not be able to overshadow effects that originate at the level of response selection-a level of processing that is involved both in selective preparation and the generation of the flanker effect.

In Experiments 1 and 2, we studied the modulation of the effects of response conflict in the flanker task by unreliable response cues. In Experiment 1, with two stimuli and two responses, there were conditions with identical, neutral, and incompatible flankers. In Experiment 2 , with four stimuli and two responses, there were conditions with identical, compatible, and incompatible flankers. In Experiment 3, we assessed the effects of fully reliable response cues as compared to a condition with neutral cues. Flankers were identical, neutral, and incompatible as in Experiment 1.

In all three experiments, we expected an effect of response cues on overall performance: RT should be shorter and error rate lower when the cued response is required than when the uncued response is required. This would confirm that the response cues indeed served to induce selective response preparation. More importantly, in the first two experiments with unreliable response cues, we expected a reduction of the flanker effect when the prepared response is required as compared to when the unprepared response is required; in the third experiment with fully reliable response cues, we expected a reduction (or even disappearance) of the flanker effect as compared to a condition with neutral cues.

Selective response preparation can result from automatic (bottomup) or controlled (top-down) processing, or both. Automatic response preparation would manifest as stimulus-driven preactivation of a response code. Controlled response preparation would manifest as preactivation driven by the deliberate expectation that a particular response is more likely than other responses to the next stimulus. The present experiments do not distinguish between automatic and controlled modes of preparation. However, we can assume that automatic pre-activation of responses is-at least partly-responsible for the response preparation observed in our experiments because we used arrowheads as response cues and because some evidence suggests that arrowheads can automatically activate a spatially compatible response (e.g., Eimer, 1995; but see Verleger, Vollmer, Wauschkuhn, van der Lubbe, \& Wascher, 2000). As the hypothesis of smaller effects of response conflict on prepared than on unprepared responses is based on 
different levels of response activation, it should be insensitive to the route by which response codes are activated and thus hold both for automatic and controlled processing of response cues.

\section{EXPERIMENT 1}

In Experiment 1, we used a two-choice task with two target stimuli and two responses. An unreliable response cue preceded each stimulus display: The cue correctly predicted the next response in $75 \%$ of the trials and incorrectly in $25 \%$. There were also trials with neutral cues that provided no information on the forthcoming response. Informative cues and neutral cues were presented in separate blocks of trials. The flankers were identical, neutral, or incompatible.

\section{Methods}

\section{PARTICIPANTS}

Twenty volunteers ( 16 female, 4 male) with a mean age of 24 years (range of 19 - 30 years) participated in Experiment 1. Participants gave informed consent before the experiment and received course credit for participation. All participants were naïve with respect to the purpose of the study and reported normal or corrected-to-normal visual acuity.

\section{APPARATUS AND STIMULI}

Participants sat in front of a 17 in. monitor, with an unconstrained viewing distance of approximately $50 \mathrm{~cm}$. All visual stimuli appeared in white $\left(\sim 75 \mathrm{~cd} / \mathrm{m}^{2}\right)$ on a black background $\left(\sim 0.5 \mathrm{~cd} / \mathrm{m}^{2}\right)$ at screen center. The response cues consisted of two arrowheads pointing to the same side $(<<$ or $>>)$, and the neutral cues consisted of two arrowheads pointing in opposite directions $(><$ or $<>)$. The stimulus displays contained a string of five capital letters: The central letter was the target and the lateral letters were the flankers. The four flankers were identical in each display. The letters $A$ and $B$ could occur as target and as flankers; the letter $C$ occurred as a (neutral) flanker only. The cues and the letters were presented in Arial font with a size of 36 . Hence, letters were on average $9 \mathrm{~mm}$ high and $8 \mathrm{~mm}$ wide. The distance between the target and each flanker, measured from stimulus center to stimulus center, was $1 \mathrm{~cm}\left(\sim 1.15^{\circ}\right)$. The mapping of target letters to response keys was counterbalanced across participants. Half of the participants pressed the left Control key of a standard keyboard to the target letter $A$ and the right Control key to the target letter $B$; the other half of the participants received the opposite mapping.

\section{PROCEDURE}

At the beginning of the experiment, instructions were presented on the monitor describing the task, the mapping of target letters to response keys, and the sequence of events in a trial. Instructions also informed participants about the cues and their validity. Then, participants performed six blocks with neutral cues and six blocks with response cues in alternating order. The first block of each type served as practice and was not further analyzed. Blocks with neutral cues consisted of two warm-up trials and 36 experimental trials in random order (two target stimuli $\times$ three flanker stimuli $\times$ six repetitions). Blocks with response cues consisted of two warm-up trials and 48 experimental trials. In the latter blocks, each of the six possible displays was presented six times with a valid response cue and two times with an invalid response cue. Hence, the response cues were valid in $75 \%$ of the trials and invalid in $25 \%$ of the trials. The sequence of cues and displays was random. Participants could take a rest between blocks and started the next block at leisure. Before each block, participants were informed whether the forthcoming block would be one with neutral cues or response cues. The whole experiment took about 30 minutes.

Each experimental trial started with a blank screen for $500 \mathrm{~ms}$, after which the cue was presented for $500 \mathrm{~ms}$, followed by a variable blank screen period of either 400,600 , or $800 \mathrm{~ms}$ duration. Then, the stimulus display was presented for $500 \mathrm{~ms}$, followed by a blank screen for $1,500 \mathrm{~ms}$. Hence, the stimulus-onset asynchrony (SOA) between the cue and the target stimulus display varied between 900 and 1,300 ms. Beginning with the onset of the cue, keypresses were monitored. RT was measured from onset of the stimulus display until the first keypress. If a wrong key was pressed, a response occurred between cue onset and $100 \mathrm{~ms}$ after target onset (i.e., target RT was shorter than $100 \mathrm{~ms}$ ), or target RT was longer than $1,100 \mathrm{~ms}$, a corresponding error message was shown for $2 \mathrm{~s}$ in red color (Arial font, font size 36). Otherwise the next trial started immediately.

\section{DESIGN AND DATA ANALYSIS}

The experiment had a $3 \times 3$ within-participant design. The first factor was Cueing Condition, with the levels valid, invalid, and neutral. The second factor was Target-Flanker Relation, with the levels identical, neutral, and incompatible.

Trials in which target RT was smaller than 100 ms (including responses between cue onset and target onset; $M=0.27 \%, S D=0.49$ ) or longer than $1,100 \mathrm{~ms}$ (less than $1 \%$ of trials) were discarded. Individual mean RTs of correct trials as well as individual error percentages (i.e., the percentages of wrong keypresses) were subjected to two-way ANOVAs, with Cueing Condition and Target-Flanker Relation as within-participant factors. If necessary, the degrees of freedom of the $F$ tests were Greenhouse-Geisser corrected. Partial eta squared and Cohen's $d$ are given as effect-size estimates. Two-tailed $t$ tests were used for planned comparisons between conditions.

The neutral cueing condition was mainly introduced for checking the typical benefits and costs of valid and invalid cues, respectively. For decomposition of the expected two-way interaction, namely, the smaller flanker effect for cued than for uncued responses, we compared the flanker effects between valid and invalid cueing conditions. This comparison should provide the most power and is typically used in related studies on the Simon effect.

\section{Results and Discussion}

Figure 1 shows the group means of the individual mean RTs. The core finding is the absence of the two-way interaction of cueing condition and target-flanker relation, $F(4,76)=0.328, M S E=197.381, p=.858$, $\eta_{\mathrm{p}}{ }^{2}=.017$. Thus, there was no indication of a modulation of the flanker 


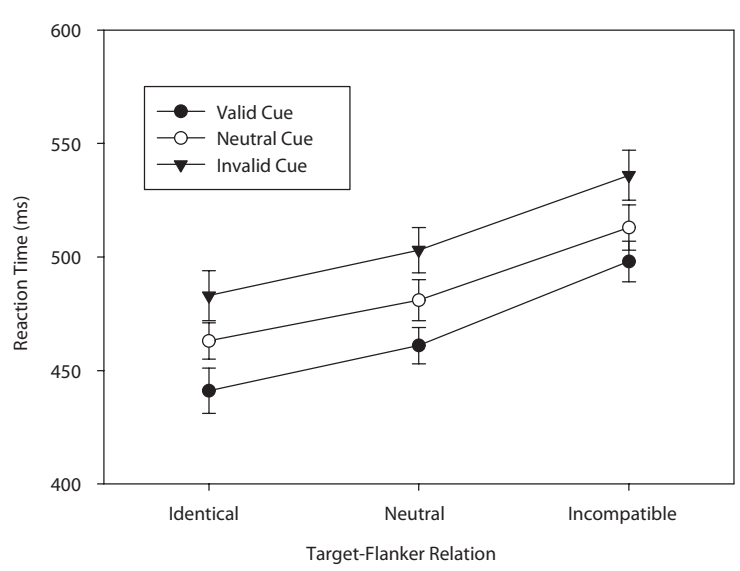

FIGURE 1.

RTs observed in Experiment 1 as a function of cueing condition (valid, neutral, invalid) and the target-flanker relation (identical, neutral, incompatible). Error bars represent standard errors between participants.

effect on RT by the response cues and no decomposition of the interaction was performed.

In contrast to the interaction, both the main effects of cueing condition, $F(2,38)=26.146, M S E=958.749, p<.001, \eta_{p}{ }^{2}=.579$, and of target-flanker relation were significant, $F(2,38)=127.125, M S E=$ $345.099, p<.001, \eta_{\mathrm{p}}{ }^{2}=.870$. Pair-wise comparisons of the different cueing conditions showed that valid cues $(M=467 \mathrm{~ms}, S D=39)$ produced shorter RTs than neutral cues $(M=486 \mathrm{~ms}, S D=40), t(19)=3.572, p<$ $.010, d=0.799$, and that neutral cues produced shorter RTs than invalid cues $(M=506 \mathrm{~ms}, S D=44), \mathrm{t}(19)=4.086, p<.010, d=0.914$. Pair-wise comparisons of the different flanker conditions revealed that identical flankers $(M=456 \mathrm{~ms}, S D=38)$ produced shorter RTs than neutral flankers $(M=475 \mathrm{~ms}, S D=37), t(19)=8.015, p<.001, d=1.792$, and that neutral flankers produced shorter RTs than incompatible flankers $(M=509 \mathrm{~ms}, S D=42), t(19)=11.181, p<.001, d=2.500$.

The group means of the individual error percentages are shown in Table 1. In contrast to RT, the two-way interaction was significant, $F(2.119,40.267)=4.736, M S E=28.985, p=.013, \eta_{\mathrm{p}}{ }^{2}=.200$. For a more detailed analysis, we compared the effects of cueing condition (valid vs. invalid), first on the difference between identical and neutral flanker conditions, and then on the difference between neutral and incompatible flanker conditions. The two-way interaction was not significant in the first of these analyses, $F(1,19)=0.026, M S E=12.155, p=.874, \eta_{\mathrm{p}}{ }^{2}$ $=.001$, but in the second one, $F(1,19)=8.874, M S E=21.425, p<.010$, $\eta_{\mathrm{p}}{ }^{2}=.318:$ The effect of response conflict on errors was amplified with invalid cues ( $8.7 \%$ instead of $2.6 \%$ with valid cues).

In addition to the interaction, both the main effects of cueing condition, $F(1.014,19.263)=9.513, M S E=91.649, p<.010, \eta_{\mathrm{p}}{ }^{2}=.334$, and of target-flanker relation were significant, $F(1.245,23.652)=19.700$, $M S E=50.688, p<.001, \eta_{\mathrm{p}}{ }^{2}=.509$. Pair-wise comparisons of the cueing conditions showed that valid cues $(M=1.944, S D=1.524)$ produced lower error rates than neutral cues $(M=3.001, S D=1.656), t(19)=$ $5.146, p<.001, d=1.151$, and neutral cues produced lower error rates
TABLE 1.

Percentages of Wrong Keypresses Observed in Experiment 1 as a Function of Cueing Condition and Target-Flanker Relation

\begin{tabular}{lccc}
\hline & \multicolumn{3}{c}{ Target-Flanker Relation } \\
& Identical & Neutral & Incompatible \\
\hline Valid Cue & $0.583(0.979)$ & $1.333(1.761)$ & $3.917(3.432)$ \\
Neutral Cue & $1.167(1.441)$ & $1.917(1.556)$ & $5.917(4.409)$ \\
Invalid Cue & $3.501(4.323)$ & $4.501(6.863)$ & $13.250(14.075)$ \\
\hline
\end{tabular}

Note. Standard deviations are given in brackets.

than invalid cues $(M=7.083, S D=7.392), t(19)=2.828, p<.050, d$ $=.632$. Pair-wise comparisons of the flanker conditions revealed that identical flankers $(M=1.250, S D=0.894)$ produced lower error rates than neutral flankers $(\mathrm{M}=2.036, \mathrm{SD}=1.471), t(19)=2.604, \mathrm{p}<.050$, $d=0.583$, and neutral flankers produced lower error rates than incompatible flankers $(M=6.107, S D=4.574), t(19)=4.728, p<.001, d=$ 1.057 .

When compared to a neutral condition without response cues, valid response cues facilitated activation of the correct response, whereas invalid response cues delayed the response. In addition, error percentages with valid response cues were smaller than in the neutral condition, and with invalid response cues they were larger. This pattern of results is the one to be expected for preactivation of the cued responses. Concerning the modulation of the effects of response conflict by response preparation, Experiment 1 produced mixed results. Whereas mean RT revealed no evidence of such a modulation, it appeared in the error percentages. In particular, the effect of response conflict as measured by the difference in error rates between conditions with neutral and incompatible flankers - was larger with invalid than with valid response cues.

\section{EXPERIMENT 2}

In order to test the robustness of the results of Experiment 1, we made several methodological changes in Experiment 2. We used a choice task with four target stimuli and two responses: Letters $A$ and $B$ were mapped on a left-hand response, and $C$ and $D$ were mapped on a righthand response. The target-flanker relations were identical, compatible, and incompatible. Instead of two flankers on each side of the target, there was only one. There was no condition with neutral cues, and there were no trials with neutral flankers.

\section{Methods}

\section{PARTICIPANTS}

Thirty volunteers ( 25 female, 5 male) with a mean age of 23 years (range of 19 - 32 years) participated in Experiment 2. Participants gave informed consent before the experiment and received course credit for participation. All participants were naïve with respect to the purpose of the study and reported normal or corrected-to-normal visual acuity. 


\section{APPARATUS AND STIMULI}

Participants sat in front of a 17 in. monitor, with an unconstrained viewing distance of approximately $50 \mathrm{~cm}$. All visual stimuli appeared in white $\left(\sim 80 \mathrm{~cd} / \mathrm{m}^{2}\right)$ on a black background $\left(\sim 0.5 \mathrm{~cd} / \mathrm{m}^{2}\right)$ at screen center. The response cues were two arrowheads that both pointed to the left $(<<)$ or to the right $(>>)$. The stimulus displays contained a string of three capital letters. The letters used were $A, B, C$, and $D$. The central letter was the target stimulus. The lateral letters were the flankers which were always identical. The cues and the letters were presented in Arial font with the size of 36 . Hence, letters were, on average, $9 \mathrm{~mm}$ high and $8 \mathrm{~mm}$ wide. The distance between the target and each flanker, measured from stimulus center to stimulus center, was $12 \mathrm{~mm}\left(\sim 1.4^{\circ}\right)$. Participants responded by pressing the Left Arrow key (to the target letter $A$ or $B$ ) or the Right Arrow key (to the target letter $C$ or $D$ ) with the index finger of the left and right hand, respectively.

\section{PROCEDURE}

At the beginning of the experiment, task instructions were presented on the monitor, the mapping of target letters to response keys, and the sequence of events in a trial. Instructions also informed participants about the cues and their validity. Then, participants performed a practice block and five experimental blocks of 64 trials each. The number of trials in each block resulted from presenting each combination of four target letters and four flanker letters four times in random order. In $75 \%$ of the trials, a valid response cue preceded the stimulus display, and in $25 \%$ - an invalid response cue. Participants could take a rest between blocks and started the next block at leisure. The whole experiment took about 30 minutes.

Each experimental trial started with a blank screen for $500 \mathrm{~ms}$, after which the cues were presented for $1 \mathrm{~s}$, followed by another blank screen for $500 \mathrm{~ms}$. Then, the stimulus display was presented until a key was pressed or for a maximal duration of $3 \mathrm{~s}$. Beginning with the onset of the stimulus display, keypresses were monitored and RT was measured. If a wrong key was pressed or if no response had occurred during stimulus presentation, a corresponding error message was shown for $1,500 \mathrm{~ms}$ in red color (Courier font, font size 24). Otherwise the next trial started immediately.

\section{DESIGN AND DATA ANALYSIS.}

The experiment had a $2 \times 3$ within-participant design. The first factor was Cueing Condition (valid vs. invalid). The second factor was Target-Flanker Relation. The flankers were either identical with the target (AAA, BBB, CCC, DDD), compatible (ABA, BAB, CDC, DCD), or incompatible (ACA, ADA, BCB, BDB, CAC, DAD, CBC, DBD).

Trials with RT below $100 \mathrm{~ms}(M=0.02 \%, S D=0.11)$ or above $1,500 \mathrm{~ms}$ (less than $1 \%$ of trials) were discarded. Individual mean RTs of correct trials and individual error percentages (i.e., percentages of wrong keypresses) were subjected to two-way ANOVAs, with Cueing Condition and Target-Flanker Relation as within-participant factors. If necessary, the degrees of freedom of the $F$ tests were GreenhouseGeisser corrected. Partial eta squared and Cohen's $d$ are given as effectsize estimates. Two-tailed $t$ tests were used for planned comparisons.

\section{Results and Discussion}

Figure 2 shows the group means of the individual mean RTs. As in Experiment 1, the two-way interaction of cueing condition and targetflanker relation was not significant, $F(2,58)=0.658, M S E=454.302 p$ $=.522, \eta_{\mathrm{p}}{ }^{2}=.022$. Again, there was no indication of a modulation of the flanker effect on RT by the cueing condition.

In contrast to the interaction, both the main effects of cueing condition, $F(1,29)=24.633, M S E=1183.401, p<.001, \eta_{\mathrm{p}}{ }^{2}=.459$, and of target-flanker relation were significant, $F(2,58)=25.815, M S E=$ 425.550, $p<.001, \eta_{\mathrm{p}}{ }^{2}=.471$. RT with valid cues $(M=510 \mathrm{~ms}, S D=$ $101)$ was faster than with invalid cues $(M=536, S D=98)$. With respect to flanker conditions, pairwise comparisons showed that identical flankers $(M=504 \mathrm{~ms}, S D=97)$ produced shorter RTs than compatible flankers $(M=513 \mathrm{~ms}, S D=98), t(29)=2.828, p<.010, d=0.516$, and compatible flankers produced shorter RTs than incompatible flankers $(M=532 \mathrm{~ms}, S D=101), t(29)=7.022, p<.001, d=1.282$.

The group means of the individual error percentages are shown in Table 2. In contrast to Experiment 1, the two-way interaction of cueing condition and target-flanker relation was not significant, $F(2,58)=$ $0.154, M S E=10.052, p=.858, \eta_{\mathrm{p}}{ }^{2}=.005$. The effect of response conflict on errors-that is, the difference between incompatible and compatible conditions, was only slightly larger with invalid than with valid cues (2.2\% vs. $1.6 \%)$.

As in Experiment 1, both the main effects of cueing condition, $F(1$, 29) $=9.703, M S E=28.135, p<.010, \eta_{\mathrm{p}}{ }^{2}=.251$, and of target-flanker relation were significant, $F(2,58)=3.556, M S E=17.150, p<.050, \eta_{\mathrm{p}}{ }^{2}=$ .109 , for error rates. Error rates were lower with valid cues $(M=2.537$, $S D=3.355)$ than with invalid cues $(M=5.000, S D=6.418)$. Regarding the flanker conditions, pairwise comparisons revealed no difference between the condition with identical flankers $(M=2.792, S D=3.020)$ and the condition with compatible flankers $(M=2.458, S D=2.871)$, $t(29)=0.812, p=.423, d=0.148$, but error rate was lower with compat-

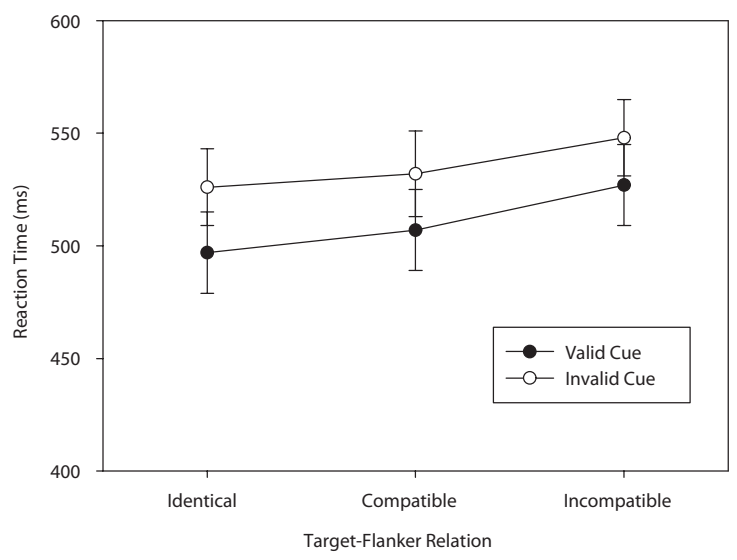

FIGURE 2.

RTs observed in Experiment 2 as a function of cueing condition (valid, invalid) and the target-flanker relation (identical, compatible, incompatible). Error bars represent standard errors between participants. 


\section{TABLE 2.}

Percentages of Wrong Keypresses Observed in Experiment 2

as a Function of Cueing Condition and Target-Flanker Relation

\begin{tabular}{lccc}
\hline & \multicolumn{3}{c}{ Target-Flanker Relation } \\
& Identical & Compatible & Incompatible \\
\hline Valid Cue & $2.222(2.814)$ & $1.889(2.504)$ & $3.500(4.746)$ \\
Invalid Cue & $4.500(5.145)$ & $4.167(6.576)$ & $6.333(7.535)$ \\
\hline
\end{tabular}

Note. Standard deviations are given in brackets.

ible flankers than with incompatible flankers $(M=4.208, S D=4.869)$, $t(29)=2.489, p<.050, d=0.454$.

The results of Experiment 2 revealed a strong effect of response cueing on performance: Performance was better with valid than with invalid response cues, both in terms of RT and accuracy. Hence, participants used the cues for preparing the cued response. In spite of the clear effect of response cues on selective preparation of the cued response, a modulation of the effects of response conflict by response preparation was absent both in RT and in error rate. The two-way interaction of cueing condition and target-flanker relation on error rate was numerically much smaller than in Experiment 1 and statistically not significant. This failure to replicate the finding of Experiment 1 cannot be attributed to a lack of statistical power. A power analysis with G-Power software (Faul, Erdfelder, Lang, \& Buchner, 2007) revealed that Experiment 2 had sufficient power $(1-\beta=.841)$ for detecting an effect of intermediate size $(d=0.25)$.

\section{EXPERIMENT 3}

In Experiment 3, we used the same two-choice task as in Experiment 1 with two target stimuli, three flankers, and two responses. In the response-cueing condition, the cue was always valid, and in the neutralcue condition, in separate blocks of trials, the cues were not predictive of the next correct response. As in Experiment 1, the interval between the cue and the stimulus display was varied randomly, so that participants were prevented from responding with a constant delay after cue presentation without attending to the display.

\section{Methods}

\section{PARTICIPANTS}

Sixteen volunteers ( 9 female, 7 male) with a mean age of 24 years (range of $21-29$ years) participated in Experiment 3. Participants gave informed consent before the experiment and received course credit for participation. All participants were naive with respect to the purpose of the study and reported normal or corrected-to-normal visual acuity.

\section{APPARATUS AND PROCEDURE}

Apparatus, stimuli, and procedure were the same as in Experiment 1 , with the exception that only valid response cues were presented (in addition to neutral cues in separate blocks of trials). There were 36 trials in each experimental block in Experiment 3.

\section{DESIGN AND DATA ANALYSIS}

The experiment had a $2 \times 3$ within-participant design. The first factor was Cueing Condition (valid vs. neutral). The second factor was Target-Flanker Relation (identical, neutral, and incompatible).

Trials in which target RT was smaller than 100 ms (including responses between cue onset and target onset; $M=1.13 \%, S D=1.09$ ) or above $1,100 \mathrm{~ms}$ (less than $1 \%$ of trials) were discarded. Individual mean RTs of correct trials and error percentages (i.e., percentages of wrong keypresses) were subjected to two-way ANOVAs, with Cueing Condition and Target-Flanker Relation as within-participant factors. If necessary, the degrees of freedom of the $F$ tests were GreenhouseGeisser corrected. Partial eta squared and Cohen's $d$ are given as effectsize estimates. Two-tailed $t$ tests were used for planned comparisons between conditions.

\section{Results and Discussion}

Figure 3 shows the group means of the individual mean RTs. Different from Experiments 1 and 2, the two-way interaction of cueing condition and target-flanker relation was significant, $F(2,28)=29.637, M S E$ $=160.356, p<.001, \eta_{\mathrm{p}}{ }^{2}=.679$. This interaction simply reflects the fact that the target-flanker relation had an effect with neutral response cues, $F(2,28)=48.881, M S E=234.887, p<.001, \eta_{\mathrm{p}}{ }^{2}=.777$, but not with always valid response cues, $F(2,28)=2.219, M S E=116.169, p$ $=.127, \eta_{\mathrm{p}}{ }^{2}=.137$. In addition to the interaction, the main effects of cueing condition, $F(1,14)=73.895, M S E=6841.743, p<.001, \eta_{p}{ }^{2}=$ .841 , and of target-flanker relation were significant, $F(2,28)=36.638$, $M S E=190.699, p<.001, \eta_{\mathrm{p}}{ }^{2}=.724$. When compared to the results of Experiment 1, the three-way interaction of Experiment $(2) \times$ Cueing Condition (2) $\times$ Target-Flanker-Relation (3) was significant for RTs, $F(2,66)=29.11, M S E=121.53, p<.001, \eta_{\mathrm{p}}{ }^{2}=.469$. The three-way interaction reflects the fact that valid cueing reduced the flanker effect in Experiment 3 (see Figure 3) but not in Experiment 1 (see Figure 1).

The group means of the individual error percentages are shown in Table 3. The two-way interaction of cueing condition and target-flanker

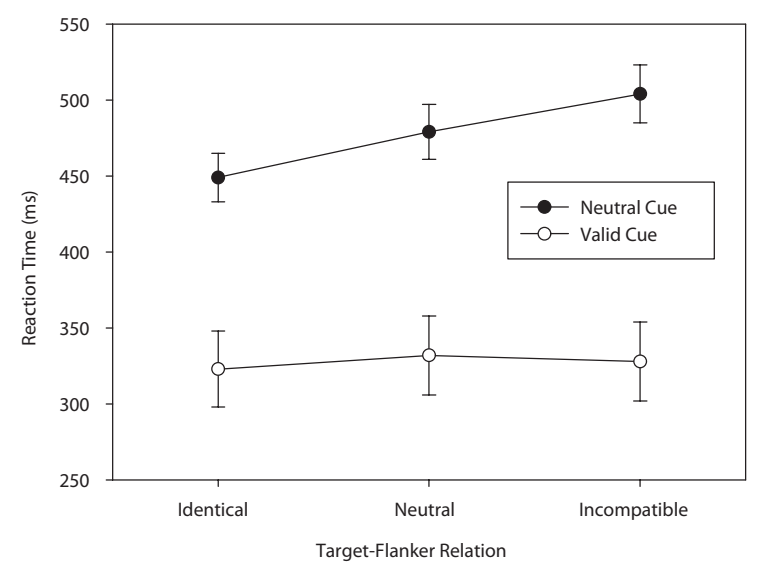

FIGURE 3.

RTs observed in Experiment 3 as a function of cueing condition (neutral, valid) and the target-flanker relation (identical, neutral, incompatible). Error bars represent standard errors between participants. 


\section{TABLE 3.}

Percentages of Wrong Keypresses Observed in Experiment 3 as a Function of Cueing Condition and Target-Flanker Relation

\begin{tabular}{lccc}
\hline & \multicolumn{3}{c}{ Target-Flanker Relation } \\
& Identical & Neutral & Incompatible \\
\hline Valid Cue & $0.222(0.586)$ & $0.778(1.526)$ & $0.556(1.744)$ \\
Neutral Cue & $1.222(1.602)$ & $2.001(2.108)$ & $6.556(4.105)$ \\
\hline
\end{tabular}

Note. Standard deviations are given in brackets.

relation was significant, $F(2,28)=16.053, M S E=3.728, p<.001, \eta_{\mathrm{p}}{ }^{2}=$ .534. As for RT, the significant interaction reflects the fact that targetflanker relation had an effect with neutral response cues, $F(2,28)=$ $18.725, M S E=6.649, p<.001, \eta_{\mathrm{p}}{ }^{2}=.572$, but not with valid response cues, $F(2,28)=0.747, M S E=1.570, p=.483, \eta_{\mathrm{p}}{ }^{2}=.051$. In addition, the main effects of cueing condition, $F(1,14)=39.640, M S E=4.264$, $p<.001, \eta_{\mathrm{p}}{ }^{2}=.739$, and of target-flanker relation were significant, $F(2$, 28) $=14.660, M S E=4.491, p<.001, \eta_{\mathrm{p}}{ }^{2}=.512$. When compared to the results of Experiment 1, the three-way interaction of Experiment (2) $\times$ Cueing Condition $(2) \times$ Target-Flanker-Relation (3) was significant for error percentages, $F(2,66)=4.14, M S E=4.17, p=.020, \eta_{\mathrm{p}}{ }^{2}=.11$, too. The three-way interaction reflects the fact that valid cueing more strongly reduced the flanker effect in Experiment 3 (see Table 3) than in Experiment 1 (see Table 1).

The results of Experiment 3 confirmed expectations. Performance was better with reliably valid response cues than without response cues, both in terms of RT and accuracy. Hence, participants consistently used the response cues for selective preparation of the indicated response. Importantly, whereas there was the usual pattern of flanker effects with neutral cues, the reliable cues eliminated the effect of the flanker stimuli on target processing. This suggests that response selection was based on the response cues, and the identity of the response stimuli (target and flankers) was neglected. Thus, with the always valid response cues, the task was essentially turned into a simple-RT task, in which the imperative signal only had to be detected to trigger production of the response that had been selected in advance.

\section{GENERAL DISCUSSION}

In all three experiments, the response cues prompted selective preparation as indicated by both faster RT and higher accuracy of the cued than of the uncued response. Contrary to expectations, however, with unreliable response cues (Experiments 1 and 2) there was no concomitant modulation of the flanker effect on RT. This finding differs not only from expectations but also from observations made for the Simon effect (Proctor et al., 1992; Verfaellie et al., 1988; Wascher \& Wolber, 2004; Wühr, 2006). For the modulation of the flanker effect on accuracy, the findings were somewhat mixed: In the first experiment, the flanker effect was smaller for prepared than for unprepared responses, mainly due to an extreme error rate for unprepared incompatible responses, but in the second experiment, the respective interaction was statisti- cally nonsignificant. We are hesitant to conclude that a modulation of the flanker effect on accuracy is absent because statistical tests of error rates tend not to be very powerful. Perhaps the modulation is generally small and unreliable as far as statistical significance is concerned.

As compared to conditions with unreliable response cues, the findings change radically with response cues that are $100 \%$ predictive of the correct response. With these cues, the flanker effect disappears both for RT and for accuracy, as it has also been found for the Simon effect (Wühr, 2006). Response selection under these conditions seems to rely (almost) fully on the response cues and no longer on the target. Similar to a simple-RT task, the response has only to be initiated upon presentation of the target, but no longer to be selected. Initiation and selection are distinct processes, as can be evidenced from the fact that they can be separated in time by way of using different stimuli for initiation and selection. With $100 \%$ valid cues, the stimulus for initiation (the target) follows the stimulus for selection (the cue), but the opposite order is possible as well (e.g., Ghez et al., 1997; Meyer, Irwin, Osman, \& Kounois, 1988).

Formally, fully valid cues are the limiting case of an increasing percentage of valid cues. Functionally, however, there might be a qualitative change with respect to the processing of the target, which turns from identification, as it is required for response selection, into detection, which is sufficient for initiation. The question remains whether there is a gradual disappearance of the flanker effect as cue validity increases from $75 \%$ to $100 \%$ and selective response preparation is progressively advanced at presentation of the target, or an abrupt and qualitative change at the transition from $99 \%$ to $100 \%$ (or perhaps at a somewhat smaller percentage), where stimulus identification turns into stimulus detection. Even without this issue being settled, however, the present findings show that the flanker effect can disappear when response selection at the time of response-signal presentation is (almost) completed.

The effects of unreliable response cues on the flanker effect and the Simon effect are clearly different. Whereas the Simon effect is larger for prepared responses than for unprepared ones, the flanker effectat least for RT-is not modulated by selective response preparation. This discrepancy is consistent with the hypothesis that the modulation of the Simon effect is caused by lateral shifts of attention (Wascher \& Wolber, 2004). Even if attentional shifts would also be present in the flanker task, they should not affect the flanker effect as long as stimulus configuration is symmetric. To note, in the Simon task it is always asymmetric.

Different from expectations, the flanker effect was not smaller for prepared than for unprepared responses, but for RT at least the effects of response preparation and flanker compatibility were additive. According to the additive-factors logic (e.g., Sternberg, 1998), the additive effects suggest that response cues and flankers affect different stages of information processing. However, there is strong independent evidence that both response cues and flankers affect the selection of responses as indicated, for example, by the lateralized readiness potential (e.g., Verleger et al., 2009; Wauschkuhn et al., 1997). 
Even from the perspective of the additive-factors logic, it cannot be excluded that additive effects may arise when two factors affect the same stage of processing. In this case, however, one would expect that additivity is restricted to certain boundary conditions. For example, the hypothesized reduction of the flanker effect might appear only at high levels of selective response preparation. This possibility is suggested by the finding of a disappearance of the flanker effect with $100 \%$ valid cues. Another boundary condition could be the decay of the influence of the flanker stimuli (e.g., C. W. Eriksen \& Schultz, 1979; Gratton et al., 1988), which has been attributed to a progressive focusing of attention on the target and a progressive neglect of the flankers. This process can be described as a continuous zooming in (cf. C. W. Eriksen \& St. James, 1986) or as a process with two discrete states (cf. Hübner, Steinhauser, \& Lehle, 2010). If the influence of the flanker stimuli decayed rapidly, their cumulative influence should be invariant across a considerable range of selective response preparation, and in the limiting case of immediate full decay, it should be absent across the full range of response preparation.

We explored these potential boundary conditions in simulations with a variant of a leaky competing accumulator model (Usher \& McClelland, 2001), a particular sequential-sampling model with lateral inhibition between response codes. The purpose of our simulations was not a data fit. Instead, we studied parametric variations to identify boundary conditions for the intuitively derived hypothesis that the flanker effect is smaller for prepared than for unprepared responses. The details are given in the Appendix. Even though the more popular diffusion model has been successfully applied to flanker tasks (White, Ratcliff, \& Starns, 2011), we chose the competitive accumulator model because it establishes a closer link between the decision between two alternatives and the activation of the corresponding responses. For the flanker task, it has been shown that, in fact, both responses are activated, albeit to different degrees (e.g., Gratton et al., 1988).

Basically, the decision between two possible responses is modelled in terms of the activation of two response codes. The theoretical activation can be thought to correspond rather directly to cortical activation of the responses, as revealed by evoked potentials or other indicators (e.g., Doyle et al., 2005; Wauschkuhn et al., 1997). In each time interval (or cycle), the activation of the two response codes is incremented by input from both the target and the flankers. The balance of the activation increments of the response codes depends on the relation between target and flankers. In addition to the deterministic components of the activation increments, there is a noise component which results in time courses of activation as illustrated in Figure 4B. Here, the activation of the correct and error responses are shown for five simulated trials, four correct choices and one incorrect choice. In the incorrect choice, the threshold (fat horizontal lines in Figure 4B) is reached for the error response first.

For the simulations, we set the stimulus-driven increments of the activations of the correct and incorrect response codes to .7 and (1-.7) for neutral flankers. The contribution of the compatible and incompatible flankers to the activation increments of the two response codes is assumed to decay in the course of successive time intervals. Initially, the value of . 7 was incremented by .15 for compatible flankers and decremented by -.15 for incompatible flankers. The decline of the flanker influence was modelled by multiplying the flanker input ( +.15 or -.15$)$ with a gating factor that declines with the passage of time, beginning at 1. For the simulations, we reduced the gating factor in each cycle to .93 , $.96, .98$, or .99 times the gating factor in the preceding cycle; the corresponding time courses are shown in Figure 4A. This reduction mimics the continuous decline of the flanker influence as a consequence of a zooming in of attention on the target. Selective response preparation was varied by setting the initial levels of the correct or incorrect response to $0, .1, \ldots, .9$, which corresponds to $0-90 \%$ of the threshold.

The results obtained with simulated trials of the competing accumulator model are shown in Figure $4 \mathrm{C}$ both for the number of cycles (which corresponds to the component of RT due to response selection) and the error rate. A preactivation of zero on the abscissa corresponds to the absence of a response cue and thus of selective preparation; positive values correspond to valid response cues and the associated preparation of the correct response, negative values correspond to invalid response cues and the associated preparation of the incorrect response. The filled data points (left ordinates) show the mean number of cycles and the error percentage in the neutral condition as a function of different preparatory states, and the outline circles (right ordinates) show the flanker effects (means of incompatible condition minus means of compatible condition). The continuous and broken lines are fifth-order polynomials fitted to the simulated data.

The results of the simulation suggest two major conclusions. First, whenever there is some uncertainty about the validity of the response cue, response preparation should be limited. The reason is the rapid increase of the error rate when the wrong response has been prepared. Second, in the range of limited preparation of the incorrect or correct response (e.g., between values of -.4 and +.4 on the abscissa of Figure $4 \mathrm{C}$, marked by broken vertical lines), the variation of the flanker effect for the number of cycles, and thus RT, is almost zero, provided the influence of the flankers decays rather rapidly. The prediction of a decreasing flanker effect with increasing preparation of the correct response is born out only for slow decay of the flanker influence. The same observation can be made for error percentages, but here the influence of preparation on the flanker effect appears already at a faster decay of the flanker influence. However, as error rates are typically not very reliable, small variations will often escape statistical significance.

The simulation confirms that additive effects of unreliable response cues and flanker compatibility are possible even when both factors affect response selection. Whether additive or interactive effects will be found depends on boundary conditions. One of these is the level of response preparation that is induced by the response cues. High levels of response preparation that turn the choice task essentially into a simple-RT task can be expected only with fully reliable response cues. Whenever there is some uncertainty, response preparation should be only weak because otherwise the error rate will approach the proportion of trials in which unprepared responses are required. A second boundary condition is the rate of decay of the influence of the conflicting stimuli or stimulus features, which may vary across tasks, pos- 
(a)

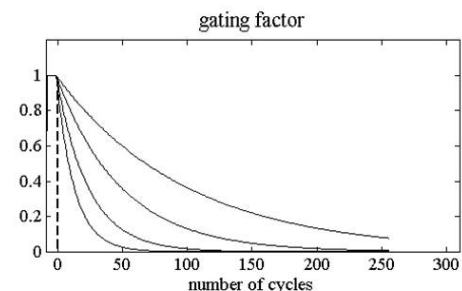

(b)

correct response
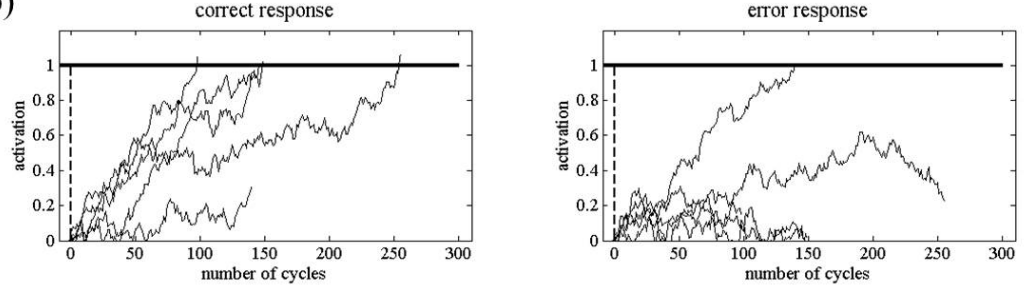

(c)
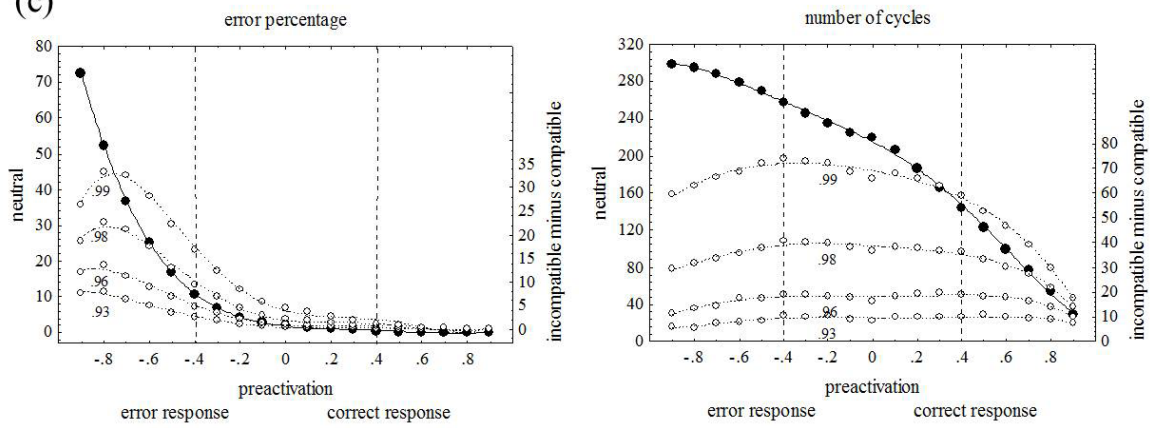

FIGURE 4.

Decay functions for the flanker influence with decay parameters of $\delta=.93, .96, .98$, and .99. (b) Example activation accumulations for the correct and error response code as a function of the number of cycles of the model. (c) Results of simulation for number of cycles and error percentage. Filled circles (left ordinate) show error percentages and number of cycles in the neutral condition, open circles show flanker effects (incompatible minus compatible condition) for decay parameters $.93, .96, .98$, and .99; continuous and dotted lines are fifth-order polynomials fitted to the data points. Error percentages and number of cycles are shown as a function of pre-activation of the correct response (positive numbers) and the incorrect response (negative numbers). Vertical broken lines mark modest levels of preparation of the correct and incorrect response.

sibly also across design features such as the proportion of trials with compatible and incompatible flankers. Finally, there may be additional boundary conditions not captured by this simulation.

\section{AUTHOR NOTE}

The authors thank Marina Annaker and Bianca Roth for running the participants and collecting the data.

\section{REFERENCES}

Ansorge, U., \& Wühr, P. (2004). A response-discrimination account of the Simon effect. Journal of Experimental Psychology: Human Perception and Performance, 30, 365-377. doi: 10.1037/00961523.30.2.365 www

Bertelson, P., \& Tisseyre, F. (1966). Choice reaction time as a function of stimulus versus response relative frequency of occurrence. Nature, 212, 1069-1070. doi: 10.1038/2121069a0 WwW

Botvinick, M. M., Braver, T. S., Barch, D. M., Carter, C. S., \& Cohen, J. D. (2001). Conflict monitoring and cognitive control. Psychological Review, 108, 624-652. doi: 10.1037/0033-295$\mathrm{X} .108 .3 .624 \underline{\mathrm{WWW}}$
Buckolz, E., Stapleton, P., \& Alain, C. (1994). Activation-interference association for finger responses in choice reaction time tasks. Acta Psychologica, 86, 1-29. doi: 10.1016/0001-6918(94)90009-4

Buhlmann, I., \& Wascher, E. (2006). Intentional pre-cueing does not influence the Simon effect. Psychological Research, 70, 117124. doi: 10.1007/s00426-004-0193-6

Cohen, J. D., Servan-Schreiber, D., \& McClelland, J. L. (1992). A parallel distributed processing approach to automaticity. American Journal of Psychology, 105, 239-269. doi: 10.2307/1423029 |WWw

Coles, M. G. H., Gratton, G., Bashore, T. R., Eriksen, C. W., \& Donchin, E. (1985). A psychophysiological investigation of the continuous flow model of human information processing. Journal of Experimental Psychology: Human Perception and Performance, 11, 529-553. doi: 10.1037/0096-1523.11.5.529 wWw

Dillon, P. J. (1966). Stimulus versus response decisions as determinants of the relative frequency effect in disjunctive reaction time performance. Journal of Experimental Psychology, 71, 321330. doi: $10.1037 / \mathrm{h} 0023003$ WWW 
Doyle, L. M. F., Yarrow, K., \& Brown, P. (2005). Lateralization of event-related beta desynchronization in the EEG during precued reaction time tasks. Clinical Neurophysiology, 116, 18791888. doi: 10.1016/j.clinph.2005.03.017 |wWW

Eimer, M. (1995). Stimulus-response compatibility and automatic response activation: Evidence from psychophysiological studies. Journal of Experimental Psychology: Human Perception and Performance, 21, 837-854. doi: 10.1037/0096-1523.21.4.837 $\underline{W W}$

Eriksen, C. W. (1995). The flankers task and response competition: A useful tool for investigating a variety of cognitive problems. Visual Cognition, 2, 101-118. doi: 10.1080/13506289508401726

Eriksen, B. A., \& Eriksen, C.W. (1974). Effects of noise letters upon the identification of a target letter in a nonsearch task. Perception \& Psychophysics, 16, 143-149. doi:10.3758/ BF03203267

Eriksen, C. W., \& Eriksen, B. A. (1979). Target redundancy in visual search: Do repetitions of the target within the display impair processing? Perception \& Psychophysics, 26, 195-205. doi: 10.3758/BF03199869

Eriksen, C. W., \& Schultz, D. W. (1979). Information processing in visual search: A continuous flow conception and experimental results. Perception \& Psychophysics, 25, 249-263. doi: 10.3758/ BF03198804 WWW

Eriksen, C. W., \& St. James, J. D. (1986). Visual attention within and around the field of focal attention: A zoom lense model. Perception \& Psychophysics, 40, 225-240. doi: 10.3758/ BF03211502 [WWw

Falkenstein, M., Hohnsbein, J., Hoormann, J., \& Kleinsorge, T. (2003). Short-term mobilisation of resources is revealed in the event-related potential. Psychophysiology, 40, 914-923. doi: 10.1111/1469-8986.00109 Www

Faul, F., Erdfelder, E., Lang, A.-G., \& Buchner, A. (2007). G*Power 3: A flexible statistical power analysis program for the social, behavioral, and biomedical sciences. Behavior Research Methods, 39, 175-191. doi: 10.3758/BF03193146 WWW

Fournier, L. R., \& Eriksen, C. W. (1990). Coactivation in the perception of redundant targets. Journal of Experimental Psychology: Human Perception and Performance, 16, 538-550. doi: 10.1037/0096-1523.16.3.538

Ghez, C., Favilla, M., Ghilardi, M. F., Gordon, J., Bermejo, R., \& Pullman, S. (1997). Discrete and continuous planning of hand movements and isometric force trajectories. Experimental Brain Research, 115, 217-233. doi: 10.1007/PL00005692 |www

Gratton, G., Coles, M. G. H., Sirevaag, E. J., Eriksen, C. W., \& Donchin, E. (1988). Pre- and poststimulus activation of response channels: A psychophysical analysis. Journal of Experimental Psychology: Human Perception and Performance, 14, 331-344. doi: 10.1037/0096-1523.14.3.331 [WWw

Heuer, H. (1982). Choice between finger movements of different and identical forms: The effect of relative signal frequency. Psychological Research, 44, 323-342. doi: 10.1007/BF00309328
Heuer, H. (1987). Visual discrimination and response programming. Psychological Research, 49, 91-98. doi: 10.1007/ BF00308673 WWW|

Hommel, B. (1997). Interactions between stimulus-stimulus congruence and stimulus-response compatibility. Psychological Research, 59, 248-260. doi: 10.1007/BF00439302

Hommel, B. (2011). The Simon effect as tool and heuristic. Acta Psychologica, 136, 189-202. doi: 10.1016/j.actpsy.2010.04.011 WWW

Hübner, R., Steinhauser, M., \& Lehle, C. (2010). A dual-stage twophase model of selective attention. Psychological Review, 117, 759-784. doi: 10.1037/a0019471 Www

Kornblum, S., Hasbroucq, T., \& Osman, A. (1990). Dimensional overlap: Cognitive basis for stimulus-response compatibility. A model and taxonomy. Psychological Review, 97, 253-270. doi: 10.1037/0033-295X.97.2.253 www

LaBerge, D., \& Tweedy, J. R. (1964). Presentation probability and choice time. Journal of Experimental Psychology, 68, 477-481. doi: 10.1037/h0048691 WwW

Laming, D. R. J. (1968). Information theory of choice-reaction times. Oxford, England: Academic Press.

Leuthold, H., Sommer, W., \& Ulrich, R. (1996). Partial advance information and response preparation: Inferences from the lateralized readiness potential. Journal of Experimental Psychology: General, 125, 307-323. doi: 10.1037/0096-3445.125.3.307 |WWW

McClelland, J. L. (1979). On the time relations of mental processes: An examination of systems of processes in cascade. Psychological Review, 86, 287-330. doi: 10.1037/0033-295X.86.4.287

Meyer, D. E., Irwin, D. E., Osman, A. M., \& Kounois, J. (1988). The dynamics of cognition and action: Mental processes inferred from speed-accuracy decomposition. Psychological Review, 95, 183-237. doi: 10.1037/0033-295X.95.2.183 Www

Miller, J. (1991). The flanker compatibility effect as a function of visual angle, attentional focus, visual transients, and perceptual load: A search for boundary conditions. Perception \& Psychophysics, 49, 270-288. doi: 10.3758/BF03214311 www

Norman, D. A., \& Shallice, T. (1986). Attention to action. Willed and automatic control of behavior. In R. J. Davidson, G. E. Schwartz, \& D. Shapiro (Eds.), Consciousness and self-regulation (pp. 1-18). New York, NY: Springer.

Proctor, R. W., Lu, C., \& Van Zandt, T. (1992). Enhancement of the Simon effect by response precuing. Acta Psychologica, 81, 5374. doi: 10.1016/0001-6918(92)90011-2 [wW]

Rosenbaum, D. A., \& Kornblum, S. (1982). A priming method for investigating the selection of motor responses. Acta Psychologica, 51, 223-243. doi: 10.1016/0001-6918(82)90036-1 Sanders, A. F. (1980). Stage analysis of reaction processes. In G. E. Stelmach \& J. Requin (Eds.), Tutorials in motor behavior (pp. 331-354). Amsterdam, the Netherlands: North-Holland.

Sanders, A. F. (1990). Issues and trends in the debate on discrete vs. continuous processing of information. Acta Psychologica, 74, 123-167. doi: 10.1016/0001-6918(90)90004-Y 
Simon, J. R. (1969). Reaction toward the source of stimulation. Journal of Experimental Psychology, 81, 1974-1976. doi: $10.1037 / \mathrm{h} 0027448 \underline{\mathrm{WWW}}$

Simon, J. R., \& Rudell, A. P. (1967). Auditory SR compatibility: The effect of an irrelevant cue on information processing. Journal of Applied Psychology, 51, 300-304. doi: 10.1037/h0020586 WWW

Smid, H. G. O. M., Mulder, G., \& Mulder, L. J. M. (1990). Selective response activation can beginn begore stimulus recognition is complete: A psychophysiological and error analysis of continuous flow. Acta Psychologica, 74, 169-201. doi: 10.1016/00016918(90)90005-Z W WW

Smith, P. L., \& Ratcliff, R. (2004). Psychology and neurobiology of simple decisions. Trends in Neurosciences, 27, 161-168. doi: 10.1016/j.tins.2004.01.006 WWW

Sternberg, S. (1969). The discovery of processing stages: Extensions of Donders' method. In W. G. Koster (Ed.), Attention and Performance II (pp. 276-315). Amsterdam, the Netherlands: North-Holland. |WWW

Sternberg, S. (1998). Discovering mental processing stages: The method of additive factors. In D. Scarborough \& S. Sternberg (Eds.), An invitation to cognitive science, Vol. 4: Methods, models, and conceptual issues (pp. 703-863). Cambridge, MA: MIT Press.

Taylor, D. A. (1977). Time course of context effects. Journal of Experimental Psychology: General, 106, 404-426. doi: 10.1037/0096-3445.106.4.404

Usher, M., \& McClelland, J. L. (2001). The time course of perceptual choice:The leaky, competing accumulator model. Psychological Review, 108, 550-592. doi: 10.1037/0033-295X.108.3.550 WWW

Verfaellie, M., Bowers, D., \& Heilman, K. M. (1988). Attentional factors in the occurrence of stimulus-response compatibility effects. Neuropsychologia, 26, 435-444. doi: 10.1016/0028-3932(88)90096-6 wWW

Verleger, R., Kuniecki, M., Möller, F., Fritzmannova, M., \& Siebner, H. R. (2009). On how the motor cortices resolve an inter-hemispheric response conflict: An event-related EEG potential-guided TMS study of the flankers task. European Journal of Neuroscience, 30, 318-326. doi: 10.1111/j.1460-9568 2009.06817.x $\overline{\mathrm{WWW}}$

Verleger, R., Vollmer, C., Wauschkuhn, B., van der Lubbe, R. J., \& Wascher, E. (2000). Dimensional overlap between arrows as cueing stimuli and responses? Evidence from contra-ipsilateral differences in EEG potentials. Cognitive Brain Research, 10, 99 109. doi: 10.1016/S0926-6410(00)00032-X WWW

Vickers, D. (1979). Decision processes in visual perception. London, England: Academic Press.

Wascher, E., \& Wolber, M. (2004). Attentional and intentional cueing in a Simon task: An EEG-based approach. Psychological

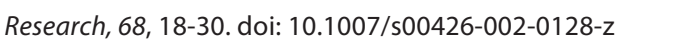

Wauschkuhn, B., Wascher, E., \& Verleger, R. (1997). Lateralised cortical activity due to preparation of saccades and finger movements: A comparative study. Electroencephalography and Clinical Neurophysiology, 102, 114-124. doi: 10.1016/S0921884X(96)96585-6 WWW

White, C. N., Ratcliff, R., \& Starns, J. J. (2011). Diffusion models of the flanker task: Discrete versus gradual attentional selection. Cognitive Psychology, 63, 210-238. doi: 10.1016/j. cogpsych.2011.08.001 WWW

Wühr, P. (2006). Response preparation modulates interference from irrelevant spatial information. Acta Psychologica, 122, 206-222. doi: 10.1016/j.actpsy.2005.12.011 WWW

Wühr, P., \& Heuer, H. (2015). The impact of response frequency on spatial stimulus-response correspondence effects. Acta Psychologica, 162, 13-19. doi: 10.1016/j.actpsy.2015.09.012 WWW

Zhang, H., Zhang, J., \& Kornblum, S. (1999). A parallel distributed processing model of stimulus-stimulus and stimulus-response compatibility. Cognitive Psychology, 38, 386-432. doi: 10.1006/ cogp.1998.0703 www

Zorzi, M., \& Umiltà, C. (1995). A computational model of the Simon effect. Psychological Research, 58, 193-205. doi: 10.1007/ BF00419634 $\overline{W W W}$

RECEIVED 27.09.2016 | ACCEPTED 08.12.2016 


\section{APPENDIX A}

The illustrative model focuses on the activation of two response codes which are self-inhibited and which inhibit each other. The process of response selection ends when one of the two activations reaches a threshold. Selective preparation is modelled by setting the initial value of the activation of one of the two response codes to larger than zero. The increase of activation in each cycle is governed by the following difference equations for the correct $\left(a_{\mathrm{c}}\right)$ and the error $\left(a_{\mathrm{e}}\right)$ response:

$$
\begin{aligned}
& \Delta a_{\mathrm{c}}(\mathrm{i})=\left[I_{\mathrm{c}}(i)-\lambda a_{\mathrm{c}}(i-1)-\beta a_{\mathrm{e}}(i-1)+N(i)\right] \Delta t \\
& \Delta a_{\mathrm{e}}(\mathrm{i})=\left[I_{\mathrm{e}}(i)-\lambda a_{\mathrm{e}}(i-1)-\beta a_{\mathrm{c}}(i-1)+N(i)\right] \Delta t \\
& \text { with input } \\
& I_{\mathrm{c}}(i)=0.5+I_{\mathrm{rel}}(i)+g(i) I_{\mathrm{irr}}(i) \\
& I_{\mathrm{e}}(i)=1-I_{\mathrm{c}}(i) \text {, }
\end{aligned}
$$

the self-inhibition gain $\lambda$, the lateral-inhibition gain $\beta$, and Gaussian noise $N$, with mean of zero and standard deviation $\sigma_{\mathrm{n}}$. The input variable $I_{\text {rel }}(i)$ is larger than 0 , and $I_{\text {irr }}(i)$ is $0,>0$, or $<0$, depending on whether the target-flanker relation is neutral, compatible, or incompatible, respectively. $I_{\text {irr }}(i)$ decays with the passage of time depending on the gating factor

$$
g(i)=\delta g(i-1), g(0)=1 .
$$

In the model, no negative activation is allowed, although $\Delta a_{\mathrm{c}}(i)$ and $\Delta a_{\mathrm{e}}(i)$ can become negative:

$$
\begin{aligned}
& a_{\mathrm{c}}(i)=\max \left[0, a_{\mathrm{c}}(i-1)+\Delta a_{\mathrm{c}}(i)\right] \\
& a_{\mathrm{e}}(i)=\max \left[0, a_{\mathrm{e}}(i-1)+\Delta a_{\mathrm{e}}(i)\right] .
\end{aligned}
$$

In the simulations, the following parameters were set to constant values: (a) Thresholds were set to 1 and $\Delta t=.01$, (b) inhibition parameters were set to $\lambda=0.3$ and $\beta=0.5$, (c) noise variance was set to $\sigma_{\mathrm{n}}=2.7$, and (d) the target input was set to $I_{\text {rel }}=0.2$. The following parameters were varied to mimic the variations of compatibility, preparation, and decay of flanker influence: (a) For compatible, neutral, and incompatible conditions, the flanker input was set to $I_{\text {irr }}=0.15,0$, and -0.15 , respectively; (b) for the variation of the decay rate from fast to slow, the decay parameter was set to $\delta=.93, .96, .98$, and .99 ; (c) for the variation of preparation of the correct response, the initial value of the its activation was set to $a_{c}(0)=0, .1, .2, .3, .4, .5, .6, .7, .8$, and .9 , with $a_{\mathrm{e}}(0)=0$, and for the preparation of the error response, the initial value of its activations was set to $a_{\mathrm{e}}(0)=0, .1, .2, .3, .4, .5, .6, .7, .8$, and .9 , with $a_{c}(0)=0$. For each parameter set, 100,000 simulated trials were run, and for each set, the percentage of error responses and the mean number of cycles of correct responses were determined. 